\title{
Impact of a structured lifestyle programme on patients with metabolic syndrome complicated by non-alcoholic fatty liver disease
}

\author{
Monica A. Konerman ${ }^{1}$ (D) | Patrick Walden ${ }^{2}$ | Megan Joseph ${ }^{2}$ | Elizabeth A. Jackson ${ }^{2}$ | \\ Anna S. Lok ${ }^{1}$ | Melvyn Rubenfire ${ }^{2}$
}

${ }^{1}$ Division of Gastroenterology and Hepatology, Department of Internal Medicine, University of Michigan, Ann Arbor, Michigan

${ }^{2}$ Division of Cardiology, Department of Internal Medicine, University of Michigan, Ann Arbor, Michigan

\section{Correspondence}

Monica A. Konerman, Division of Gastroenterology and Hepatology, University of Michigan, Ann Arbor, Ml.

Email: konerman@med.umich.edu

\section{Summary}

Background: Lifestyle interventions are first-line therapy for non-alcoholic fatty liver disease (NAFLD).

Aims: To examine the prevalence of NAFLD among participants of the University of Michigan Metabolic Fitness (MetFit) Programme and to assess the impact of this programme on weight, metabolic and liver-related parameters among patients with and without NAFLD.

Methods: Adults who completed the programme between 2008 and 2016 were included. Clinical and laboratory data were collected at enrolment, and at 12 and 24 weeks. NAFLD was defined based on liver biopsy, imaging or clinical diagnosis.

Results: The cohort ( $\mathrm{N}=403 ; 253$ 12-week, 150 24-week) consisted primarily of middle-aged (median 54 years) white (88\%) men (63\%) with severe obesity (median BMI 37.4). 47.6\% met criteria for NAFLD. At baseline, NAFLD patients were younger (52 vs 55 years), had higher weights and more metabolic derangements (higher fasting insulin and triglyceride, lower high-density lipoprotein-cholesterol). At programme completion, $30 \%$ achieved weight reduction $\geq 5 \%, 62 \%$ resolution of hypertriglyceridaemia, $33 \%$ resolution of low HDL, $27 \%$ resolution of impaired fasting glucose and $43 \%$ normalisation of alanine aminotransferase. Endpoints were unaffected by NAFLD. Longer programme duration (OR 6.7, 95\% $\mathrm{Cl} 3.6-12.3$ ) and white race (OR $3.83,95 \% \mathrm{Cl} 1.04-1.76)$ were independent predictors of $\geq 5 \%$ weight loss.

Conclusions: Nearly half of the patients referred to a structured lifestyle programme for metabolic syndrome had NAFLD. Although baseline metabolic derangements were more pronounced among NAFLD patients, the programme was equally efficacious in achieving weight loss and resolving metabolic syndrome components. Programme duration was the most important predictor of response. 


\section{INTRODUCTION}

Obesity has become a worldwide public health crisis given its prevalence and multiple associated complications including non-alcoholic fatty liver disease (NAFLD). Metabolic syndrome (MetSyn) is highly correlated with prevalent and incident NAFLD. Approximately $46 \%$ of patients with NAFLD have concomitant MetSyn, and odds of developing NAFLD are significantly higher among patients with baseline MetSyn (odds ratios [OR] 4 for men and 11.2 for women). ${ }^{1,2}$ The presence of MetSyn has been identified as a predictor for underlying non-alcoholic steatohepatitis (NASH) among patients with NAFLD, and the total number of metabolic risk factors in an individual NAFLD patient is correlated with risk of liver disease progression. ${ }^{3,4}$ First-line treatment for metabolic disease associated with obesity including NAFLD is lifestyle interventions. From a liver perspective, multiple studies have demonstrated improvement in hepatic steatosis and histologic components of NASH among patients who are able to achieve weight loss. ${ }^{5,6}$ While $10 \%$ reduction in body weight is generally required to resolve steatosis, inflammation and fibrosis, a more modest reduction (3\%-5\%) has been shown to decrease steatosis.

In clinical practice, it can be challenging for patients to implement lifestyle changes. Highly structured lifestyle programmes often have the highest efficacy for weight loss. One such programme is the University of Michigan Metabolic Fitness (MetFit) Programme. Although many regimented lifestyle programmes were initially targeted for patients with MetSyn with cardiology and endocrinology referral bases, these types of programmes represent potential therapeutic options for patients with NAFLD. The aims of this study were (a) to evaluate the prevalence of NAFLD among participants enrolled in the MetFit Program and to characterise their patterns of clinical care and (b) to assess the impact of the MetFit Program among participants with and without NAFLD and to identify predictors of achieving significant weight loss at programme completion.

\section{MATERIALS AND METHODS}

\subsection{Metabolic Fitness Program}

The University of Michigan's MetFit Program was initially designed for patients with MetSyn, with detailed description of the programme design previously described, but reviewed here. ${ }^{7}$ MetSyn was defined as having at least three of the five following variables: waist circumference of $\geq 40$ inches in men and $\geq 35$ inches in women, triglycerides (TG) $\geq 150 \mathrm{mg} / \mathrm{dL}$ or treatment targeting $\mathrm{TG}$, high-density lipoprotein-cholesterol (HDL-C) $<40 \mathrm{mg} / \mathrm{dL}$ in men and $<50 \mathrm{mg} / \mathrm{dL}$ in women, fasting glucose $\geq 100 \mathrm{mg} / \mathrm{dL}$ or diabetes, and systolic blood pressure $\geq 130 \mathrm{~mm} \mathrm{Hg}$ or diastolic blood pressure $\geq 85 \mathrm{~mm} \mathrm{Hg}$ or on treatment for hypertension (HTN). ${ }^{8}$ Indications for referral have expanded and include NAFLD with or without MetSyn. Both a 12- and 24-week programmes are offered. ${ }^{9}$
The programme was designed to maximise cost-effective and time-efficient real-world delivery of care and thus utilises an interactive small group design (rather than costly and less efficient one-onone design).

Sessions occur once per week with each session comprised of a 45-min educational lecture and a 45-min supervised exercise session. Lectures are given in small group settings (roughly 10-15 patients per session), with an interactional model that allows individual participants to discuss their personal goals and barriers and receive feedback from the instructor. Lecture topics include the following: an orientation lecture on the programme, several lectures on nutritional topics, several lectures on the impact of stress and behaviour change, dedicated lectures on exercise and lectures on clinical impact of metabolic syndrome. Nutritional lecture topics included the Mediterranean diet, reducing sodium intake, meal planning, portion control, eating healthily while dining out and interpreting food labelling to tailor to a healthy eating plan. The topics for the stress/behavioural educational lecture series included emotional barriers to lifestyle change, strategies to increase awareness of emotional needs and strategies to decrease the impact of psychosocial stressors on food choices.

Two exercise physiologists were available during exercise sessions to monitor safety, and educate and encourage participants. Aerobic exercise intensity was prescribed based on heart rate from entry graded exercise testing. Exercise intensity was prescribed based upon the symptom-limited stress test (treadmill exercise electrocardiogram) and provided as $50 \%-75 \%$ of predicted maximal heart rate and perceived exertion as moderately hard. Heart rate targets were $60 \%-85 \%$ of heart rate reserve formula (difference between resting heart rate and maximum predicted heart rate). When graded exercise testing was not indicated or not available, Borg ratings of perceived exertion scale were used. ${ }^{10}$ The Borg rating ranges from 6 (no exertion) to 20 (maximal exertion). Participants were encouraged to target exertion levels between 11 (fairly light) and 13 (somewhat hard). Some low-risk participants were allowed to exercise to intensities of 15 . BMI and central obesity were taken into account for exercise regimen on an individual, as needed basis according to participant's report to the exercise physiologists who monitored each session with alternative exercises provided based on participant-reported limitations. ${ }^{11,12}$ Participants were asked to keep a logbook of aerobic exercise equipment used and total dedicated aerobic and resistance training each day. Supervised exercise sessions include aerobic exercise (typically lasting 30-40 min) using equipment of participant choice (treadmill, bike and elliptical), exercise tubes and freeweight strength training. The remaining 5-10 min included stretching exercises. As a complement to aerobic exercise, participants engaged in a full-body strengthening programme (free weight, resistance bands or combination circuit training targeting all major muscle groups [legs, chest, back, shoulders, core, biceps and triceps]). In addition to these supervised sessions, participants were advised to engage in 150-300 min of exercise per week consisting of a combination of moderate/vigorous-intensity aerobic activity and strength/ resistance training. Each session of exercise was recommended to 
last a minimum of 20 min with a maximum of 60 min per session. In addition to this moderate- to vigorous-intensity exercise, a full-body strengthening programme (free weight, resistance bands or combination circuit training) was recommended minimum two times weekly targeting all major muscle groups. Participants had access to the exercise facility throughout the course of the programme to use for this purpose.

Dietitians provided nutritional recommendations based on a Mediterranean diet targeted towards a 12-week weight loss goal of $5 \%$ and 24 -week weight loss goal of $10 \%$ of enrolment body weight. Nutrition data were collected at entry and at completion of the programme using a food frequency assessment focusing on a Mediterranean eating pattern. As part of the initial intake evaluation, personalised energy intake guidelines were calculated by a registered dietitian, as estimated by the Mifflin-St Jeor equation based on sex, age, height, weight and activity level. ${ }^{13}$ Resting energy equivalent as determined by the Mifflin-St Jeor equation was multiplied by an activity factor of 1.3 for participants who fully participated in the exercise portion of the programme. For less active participants, an activity factor of 1.2 was recommended to estimate energy needs.

\subsection{Data collection}

Patients complete an initial 1-h assessment including a history and physical, and evaluation for barriers to participation. Potential barriers include severe orthopaedic, musculoskeletal or cardiopulmonary disease, or any other self-reported barriers that would preclude the individual from completing components of the programme. A Framingham risk assessment and the Patient Health Questionnaire-9 (PHQ-9) were completed. ${ }^{14}$ The PHQ-9 is a 9-item depression score with each item rated 0 (not at all) to 3 (nearly every day), with total scores of 5-9 representing possible depression, 10-14 high probability of depression and $>14$ high probability of major depression. Baseline height, weight, BMI, waist circumference, body composition percentage (assessed using bioelectrical impedance, Tanita Body Composition Analyzer Model TBF-310; Tanita, Arlington Heights, IL, USA), blood pressure (systolic and diastolic per American Heart Association standard protocol), glucose, insulin, total cholesterol, TG, HDL-C and low-density lipoprotein-cholesterol (LDL-C) were obtained after a 12-h fast. Haemoglobin A1c (A1c) and alanine aminotransferase (ALT) were also obtained. The baseline assessment was repeated at 12 weeks and then again at 24 weeks for those in the 24-week programme. Ethics approval was regulated by the University of Michigan Health System Institutional Review Board (HUM0045929), and all participants provided written informed consent.

\subsection{Study inclusion and exclusion criteria}

To be included in the primary analysis for this outcome study, patients had to complete the programme, as defined by attending $>75 \%$ of sessions and needed to complete the end of programme data collection for biometrics and laboratory studies.

\subsection{Definition of NAFLD and outcomes of interest}

The diagnosis of NAFLD was ascertained using a natural language processing programme (University of Michigan EMERSE) and manual chart review by a hepatologist (MAK). ${ }^{15} \mathrm{~A}$ patient was classified as meeting criteria for NAFLD if they had any of the following in the absence of significant alcohol use (defined as $>14$ drinks per week in men and $>7$ drinks per week in women; alcohol use was obtained via self-report at time of programme enrolment): evidence of steatosis or steatohepatitis on liver biopsy; imaging including ultrasound, computed tomography scan or magnetic resonance imaging noting hepatic steatosis; a clinical diagnosis code (ICD-9 or 10) for NAFLD or NASH; or a combination of the above. We also assessed proportion of patients with NAFLD referred and managed by hepatology and/or gastroenterology (GI). In addition, we analysed differences in baseline clinical characteristics and the impact of the programme between patients with and without NAFLD. Specifically, we investigated percentage weight loss and predictors of weight loss, and changes in metabolic and liver-related parameters and change in depression scores. Insulin resistance was assessed by homeostasis model assessment-estimated insulin resistance (HOMA-IR) among patients without baseline diabetes and defined as (fasting plasma insulin $\times$ fasting plasma glucose)/22.5, with a value $>2.9$ indicative of significant insulin resistance. ${ }^{16}$

\subsection{Statistical analyses}

Descriptive and bivariate analyses were performed to assess baseline characteristics and impact of the MetFit Program on outcomes of interest. Chi-squared tests and Fisher's exact tests were used for categorical variables, and $t$ tests were used for continuous variables. Variables with distributions that deviated from normality were reported by median and interquartile range (Q1, Q3) and were compared using the Kruskal-Wallis test. Intention-to-treat analysis was performed for primary outcomes of interest to account for programme dropout using multiple variable imputation. Multivariate analysis was performed to identify predictors of weight loss. Candidate covariates were assessed for inclusion into the multivariable model based on $P<0.10$ in the univariate analysis and biologic plausibility. $P \leq 0.05$ was considered statistically significant. All analyses were performed in Stata 14 (StataCorp, College Station, TX).

\section{RESULTS}

\subsection{Programme adherence and dropout}

Overall completion rate for the MetFit Program was approximately $79 \%$ (defined as percentage of participants who complete $\geq 75 \%$ of sessions). Dropout occurred mostly in the second half of the programme. Characteristics of patients who did vs those who did not complete the programme are detailed in Table S1. Overall, there was a higher proportion of females among the population of participants 
who did not complete the programme (among participants who did not complete the programme, $69 \%$ were female, whereas $37.5 \%$ of programme completers were female, $P<0.001$ ). There was a similar proportion of patients with NAFLD who dropped out vs completed the programme (41.6\% vs $47.6 \%)$. Participants who dropped out of the programme had a higher prevalence of central obesity $(95.5 \%$ vs $88.1 \%, P=0.03$ ) but a lower prevalence of hypertriglyceridaemia (42.2\% vs $54.1 \%, P=0.04$ ) and low HDL-C (38.9\% vs $57.3 \%$, $P=0.002)$. Of note, those who dropped out had higher baseline PHQ-9 depression scores (7 vs 5, $P=0.04$ ) and tended to have more extensive formal educational backgrounds. Baseline characteristics were otherwise similar between those who dropped out and those who completed the programme.

\subsection{NAFLD prevalence and care patterns}

Within this cohort, $47.6 \%$ of patients had evidence of NAFLD. Sixteen $(8.3 \%)$ met criteria based on a combination of imaging, clinical and histologic criteria, 96 (50\%) met criteria based on a combination of imaging and clinical diagnosis, $45(23.4 \%)$ by clinical diagnosis alone and 35 (18.2\%) by imaging criteria alone. Among those meeting diagnostic criteria for NAFLD, only 47 (25\%) were managed by $\mathrm{GI} /$ Hepatology for NAFLD. Of note, an additional $31 \%$ of patients with NAFLD had established care with a GI provider for other conditions, but did not have their NAFLD managed or addressed by the GI provider (Figure 1).

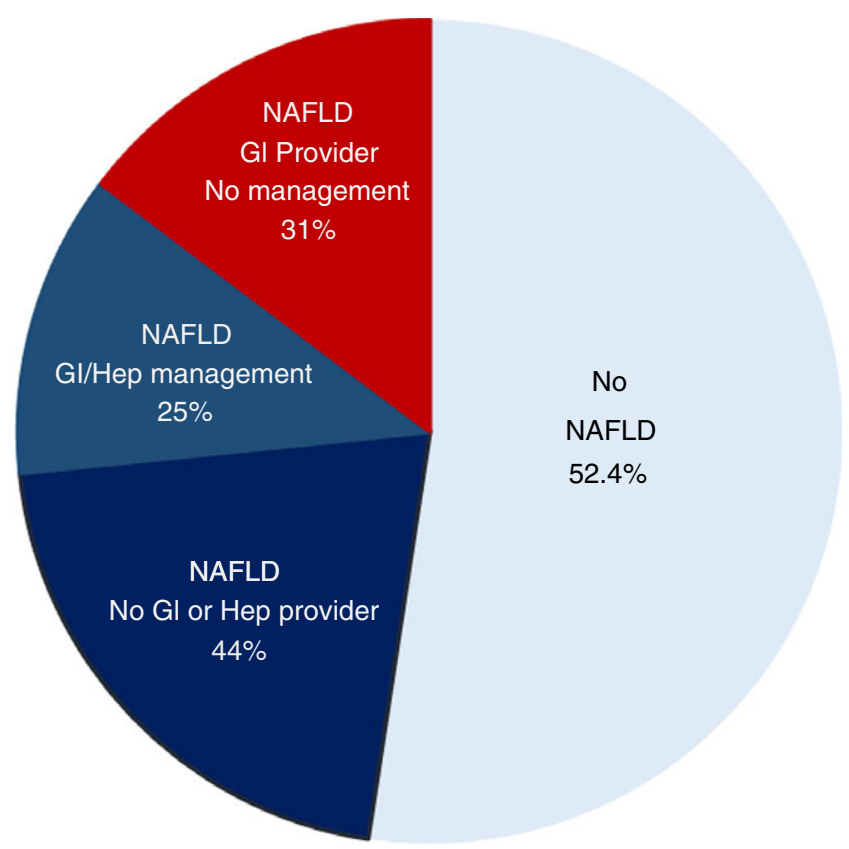

FIGURE 1 NAFLD prevalence and care patterns among participants in Metabolic Fitness Program. Pie chart depicting percentage of participants in Metabolic Fitness Program with evidence of underlying non-alcoholic fatty liver disease (NAFLD) and distribution of NAFLD patients who received specialty care for their NAFLD. GI, gastroenterology; Hep, hepatology

\section{3 | Baseline patient characteristics}

The baseline characteristics of patients are displayed in Table 1. Overall, the cohort consisted primarily of middle-aged (median 54 years) white (88.6\%) men (62.5\%) with severe obesity (median BMI 37.4). All five components of MetSyn were present in $19.9 \%$, four components in $26.9 \%$ and three components in $27.9 \%$. NAFLD patients had higher numbers of components of the MetSyn (median 4 vs $3, P=0.001$ ). At baseline, patients with NAFLD were younger (52 vs 55 years, $P=0.004$ ) and more likely to have higher weight (242 vs $227 \mathrm{lb}, P=0.01$ ) and maximum lifetime weight (250 vs $231 \mathrm{lb}, P=0.008$ ). NAFLD patients also had higher prevalence of features of the metabolic syndrome and insulin resistance, and higher prevalence of abnormal ALT. There were no significant differences in baseline characteristics between participants who completed the 12-week vs 24-week programme aside from a higher prevalence of baseline HTN in the 24-week programme (75\% vs $57.8 \%, P=0.001$ ) (Table S2). Baseline NAFLD Fibrosis Score (NFS), a noninvasive biomarker of fibrosis in NAFLD, was available in 90 (47\%) NAFLD patients. The median NFS was -0.62 (IQR -1.56 to 0.26 ), with a value of $<-1.455$ consistent with no-to-minimal fibrosis and a score of $>0.675$ consistent with advanced fibrosis. ${ }^{17}$

\subsection{Impact of programme on outcomes of interest}

Results of the impact of the MetFit Program are outlined in Table 2. At 12 weeks, a weight reduction $\geq 5 \%$ was achieved in $23 \%$ of participants and weight reduction $\geq 10 \%$ in $3.4 \%$ of participants. The programme was efficacious in eliminating components of the MetSyn, with resolution of hypertriglyceridaemia (62\%) being most common. The degree of change in triglyceride level was not significantly different based on use of statin at enrolment ( $N=168$ on statins). The distribution of number of components of the MetSyn at 12 weeks was similar among those with and without NAFLD (median $=3$ for both, $P=0.19$ ). Also of note, $36 \%$ of patients with baseline abnormal ALT had normalised ALT and median PHQ-9 score improved by 2 points. When the impact of the programme at 12 weeks was stratified by the presence of NAFLD, weight loss and improvement in metabolic parameters were similar in patients with and without NAFLD, but patients with NAFLD had a greater decrease in percentage of body fat $(-1.5$ vs $-0.9, P=0.009)$ and a larger reduction in ALT ( -5.5 vs $-2 \mathrm{U} / \mathrm{L}, P=0.004$ ) (Figure 2). Preand post-NFS was available in 36 (19\%) NAFLD patients, with a median change in NFS of -0.21 (IQR -0.73 to 0.77 ).

In the 24-week programme, weight reduction $\geq 5 \%$ was achieved in $53 \%$ of participants and weight reduction $\geq 10 \%$ in $16 \%$ of participants. At week 24, median weight loss was 12.5 lb (IQR 5-20.5), resolution of hyperTG was observed in 35\%, low HDL-C in 34\%, impaired fasting glucose in $30.6 \%$ and insulin resistance in $28.7 \%$. The distribution of number of components of the MetSyn at 24 weeks was similar among those with and without NAFLD (median $=3$ for both, $P=0.32$ ). Roughly half (54\%) of patients with an abnormal ALT had normalised their ALT. Median PHQ-9 score remained improved by 2 
TABLE 1 Baseline patient characteristics according to NAFLD diagnosis

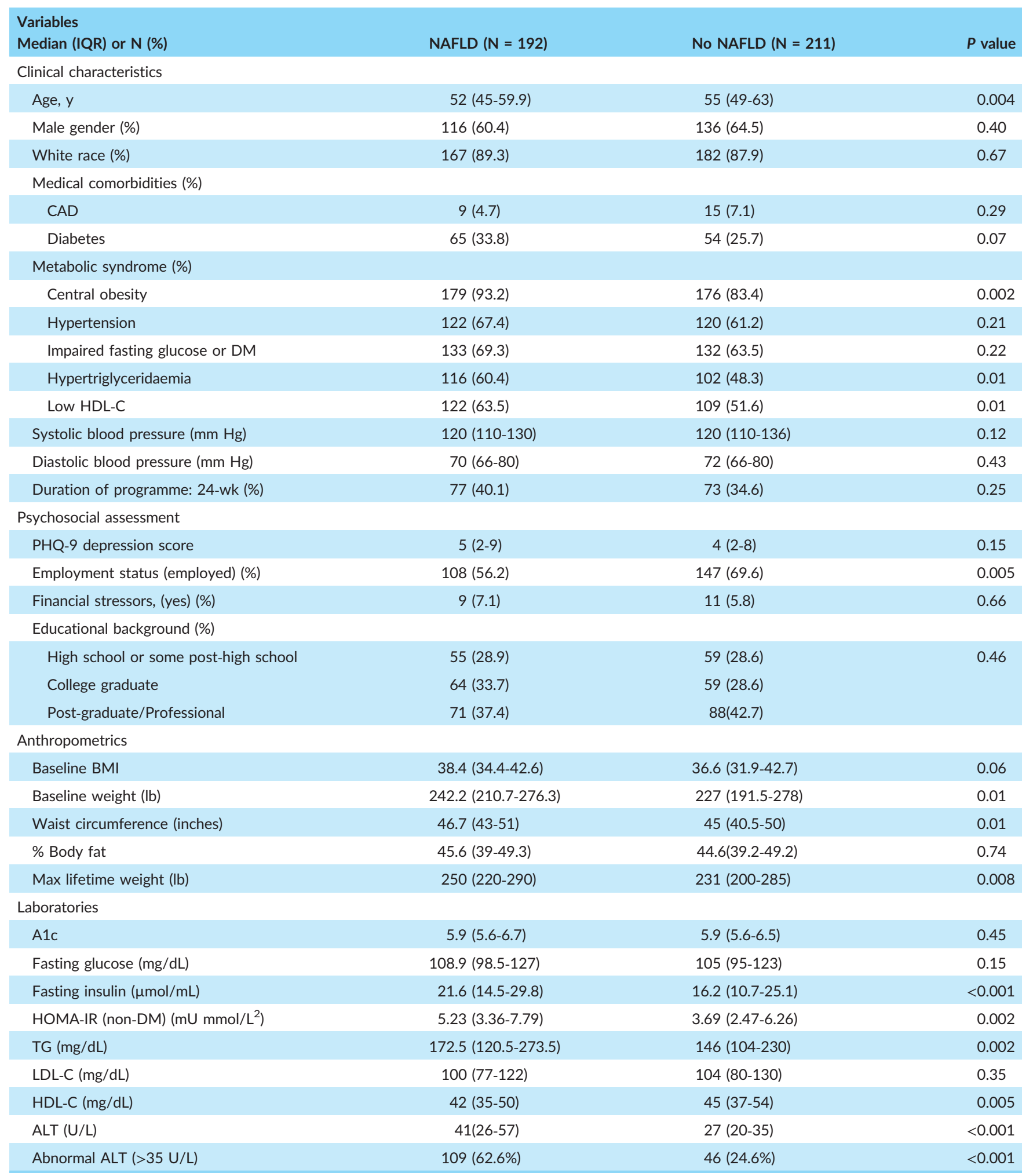

Central obesity defined as $\geq 40$ inches in men and $\geq 35$ inches in women.

Hypertension defined as SBP $>130 \mathrm{~mm} \mathrm{Hg}$, DBP $>85 \mathrm{~mm} \mathrm{Hg}$ or on antihypertensive.

Impaired fasting glucose defined as $>100 \mathrm{mg} / \mathrm{dL}$ or DM; hyperTG defined as $\geq 150 \mathrm{mg} / \mathrm{dL}$ or on treatment for triglycerides.

Low HDL-C defined as $<40 \mathrm{mg} / \mathrm{dL}$ men and $<50 \mathrm{mg} / \mathrm{dL}$ women.

ALT, alanine aminotransferase; BMI, body mass index; CAD, coronary artery disease; DM, diabetes mellitus; HDL-C, high-density lipoprotein-cholesterol; HOMA-IR, homeostasis model assessment-estimated insulin resistance; LDL-C, low-density lipoprotein-cholesterol; TG, triglycerides. 
TABLE 2 Impact of Metabolic Fitness Program on metabolic, liver-related and depression parameters stratified by NAFLD status

\begin{tabular}{|c|c|c|c|}
\hline $\begin{array}{l}\text { Change in variable from baseline to end of programme } \\
\text { Median (IQR) or } \mathrm{N}(\%)\end{array}$ & NAFLD & No NAFLD & $P$ Value \\
\hline 12-week & $N=192$ & $N=211$ & \\
\hline Weight (lb) & $-6.8(-2 ;-12.7)$ & $-5.5(-1.6 ;-10)$ & 0.08 \\
\hline$\geq 5 \%$ Reduction & $48(25 \%)$ & $44(20.9 \%)$ & 0.32 \\
\hline$\%$ Body fat & $-1.5(-0.2 ;-2.9)$ & $-0.9(0 ;-2.2)$ & 0.009 \\
\hline \multicolumn{4}{|l|}{ Features of metabolic syndrome (\%) } \\
\hline Loss of central obesity & $11(6.1)$ & $18(10.3)$ & 0.15 \\
\hline Resolution of hypertension & $9(7.6)$ & $7(5.8)$ & 0.59 \\
\hline Resolution of low HDL & $44(36.1)$ & $35(32.7)$ & 0.59 \\
\hline Systolic blood pressure $(\mathrm{mm} \mathrm{Hg})$ & $-4(-12 ;+5)$ & $-6(-18 ;+2)$ & 0.29 \\
\hline Diastolic blood pressure $(\mathrm{mm} \mathrm{Hg})$ & $-2(-8 ;+4)$ & $-3(-10 ;+2)$ & 0.35 \\
\hline A1c & $-0.2(0 ;-0.5)$ & $-0.1(0 ;-0.3)$ & 0.15 \\
\hline Fasting glucose (mg/dL) & $-5(+4 ;-14)$ & $-3(+3 ;-11)$ & 0.21 \\
\hline Insulin $(\mu \mathrm{mol} / \mathrm{mL})$ & $-2.4(+2.4 ;-9.2)$ & $-0.8(+2.4 ;-6.3)$ & 0.09 \\
\hline HOMA-IR (non-DM), (mU mmol/L²) & $-0.73(+0.44 ;-2.44)$ & $-0.16(+0.49 ;-1.49)$ & 0.17 \\
\hline $\mathrm{TG}(\mathrm{mg} / \mathrm{dL})$ & $-32.5(+10 ;-93)$ & $-22(+3 ;-57)$ & 0.28 \\
\hline LDL-C (mg/dL) & $0(+10 ;-11)$ & $-3(+9 ;-14)$ & 0.18 \\
\hline Weight (lb) (\%) & $-13.5(-3.5 ;-22.5)$ & $-11.7(-5.5 ;-18.5)$ & 0.72 \\
\hline$\geq 5 \%$ Reduction & $43(55.8)$ & $36(49.3)$ & 0.42 \\
\hline$\geq 10 \%$ Reduction & $12(15.6)$ & $12(16.4)$ & 0.88 \\
\hline Waist circumference & $-2.9(-0.7 ;-4.5)$ & $-2.5(-0.5 ;-4)$ & 0.59 \\
\hline$\%$ Body fat & $-1.9(-0.3 ;-4.1)$ & $-1.9(-0.7 ;-3.4)$ & 0.94 \\
\hline \multicolumn{4}{|l|}{ Features of metabolic syndrome (\%) } \\
\hline Loss of central obesity & $11(15.1)$ & $10(16.4)$ & 0.83 \\
\hline Resolution of hypertension & $6(10.7)$ & $3(6.3)$ & 0.44 \\
\hline $\begin{array}{l}\text { Resolution of indicators of insulin resistance } \\
\text { (reduction in fasting glucose to } \leq 100 \mathrm{mg} / \mathrm{dL} \text { ) }\end{array}$ & $13(27.1)$ & $17(34)$ & 0.46 \\
\hline Resolution of hypertriglyceridaemia & $18(35.9)$ & $14(35.9)$ & 0.89 \\
\hline Resolution of low HDL & $15(30.6)$ & $15(37.5)$ & 0.49 \\
\hline Systolic blood pressure (mm Hg) & $-3(-18 ;+6)$ & $-8(-18 ;+6)$ & 0.42 \\
\hline Diastolic blood pressure $(\mathrm{mm} \mathrm{Hg})$ & $-2(-12 ;+6)$ & $-2(-14 ;+4)$ & 0.66 \\
\hline A1c (\%) & $-0.2(0 ;-0.5)$ & $-0.1(0 ;-0.6)$ & 0.88 \\
\hline Fasting glucose $(\mathrm{mg} / \mathrm{dL})$ & $-6(+4 ;-17)$ & $-4(+3 ;-11)$ & 0.58 \\
\hline Insulin $(\mu \mathrm{mol} / \mathrm{mL})$ & $-2.5(-0.5 ;-9.1)$ & $-4.9(-1.5 ;-9.8)$ & 0.06 \\
\hline HOMA-IR (non-DM) (mU mmol/ $\left.\mathrm{L}^{2}\right)$ & $-0.72(+0.35 ;-2.21)$ & $-1.38(-0.30 ;-3.15)$ & 0.06 \\
\hline
\end{tabular}


TABLE 2 (Continued)

\begin{tabular}{|c|c|c|c|}
\hline $\begin{array}{l}\text { Change in variable from baseli } \\
\text { Median (IQR) or } N(\%)\end{array}$ & NAFLD & No NAFLD & $P$ Value \\
\hline TG (mg/dL) & $-45(-9 ;-113)$ & $-23(+3 ;-58)$ & 0.05 \\
\hline $\mathrm{HDL}-\mathrm{C}(\mathrm{mg} / \mathrm{dL})$ & $+1(+6 ;-4)$ & $0(+3 ;-3)$ & 0.51 \\
\hline ALT (U/L) & $-11(-0.5 ;-28.5)$ & $-3(+1 ;-9)$ & 0.003 \\
\hline
\end{tabular}

Central obesity defined as $\geq 40$ inches in men and $\geq 35$ inches in women; hypertension defined as SBP $>130 \mathrm{~mm} \mathrm{Hg}$, DBP $>85 \mathrm{~mm} \mathrm{Hg}$ or on antihypertensive. Impaired fasting glucose defined as $>100 \mathrm{mg} / \mathrm{dL}$ or DM; hyperTG defined as $\geq 150 \mathrm{mg} / \mathrm{dL}$ or on treatment for triglycerides; low HDL-C defined as $<40 \mathrm{mg} / \mathrm{dL}$ men and $<50 \mathrm{mg} / \mathrm{dL}$ women.

ALT, alanine aminotransferase; BMI, body mass index; HDL-C, high-density lipoprotein-cholesterol; HOMA-IR, homeostasis model assessment-estimated insulin resistance; LDL-C, low-density lipoprotein-cholesterol; TG, triglycerides.
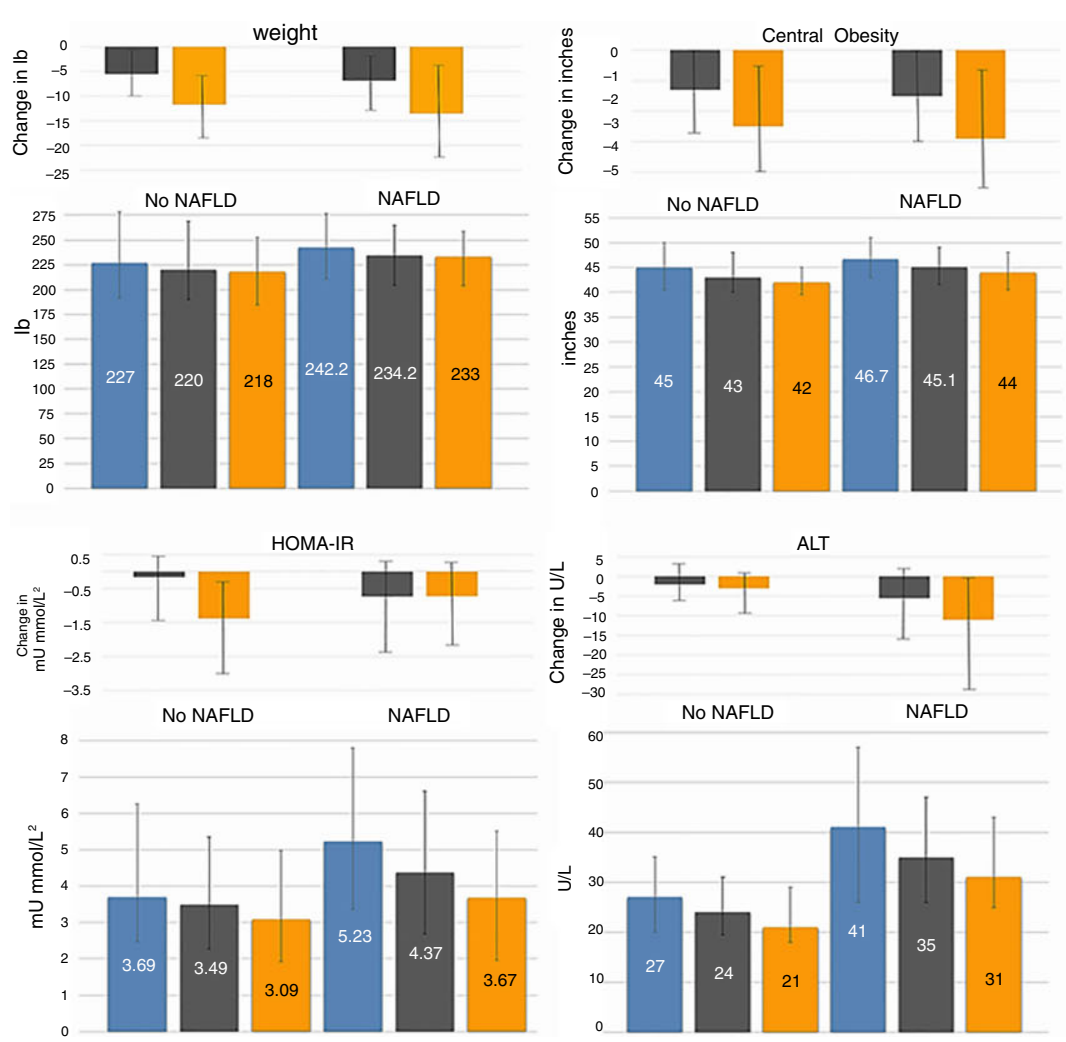
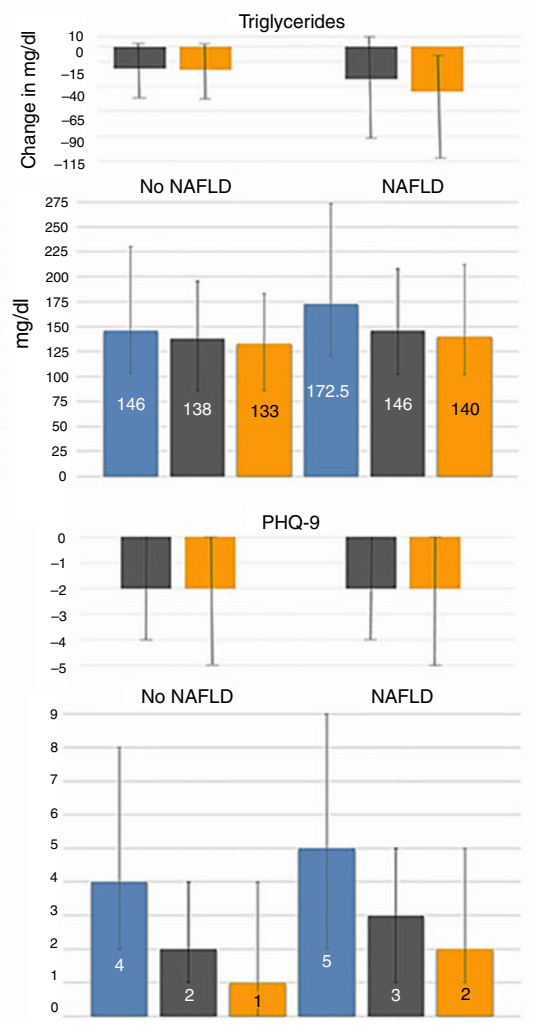

FIGURE 2 Changes in metabolic, liver and depression parameters based on underlying NAFLD and programme duration. Bar charts show changes in weight, central obesity, triglycerides (TG), homeostasis model assessment-estimated insulin resistance (HOMA-IR), alanine aminotransferase (ALT) and Patient Health Questionnaire-9 (PHQ-9) from baseline to 12 and 24 weeks of the Metabolic Fitness Program, stratified by non-alcoholic fatty liver disease (NAFLD) status. Improvements were similar in patients with and without NAFLD, but patients with NAFLD had a greater decrease in TG and a larger reduction in ALT. Values represent median with brackets representing interquartile ranges

points compared to baseline. Weight loss and improvement in metabolic parameters were similar in patients with and without NAFLD, but patients with NAFLD were noted to have a greater decrease in TG $(-45 \mathrm{mg} / \mathrm{dL}$ vs $-23 \mathrm{mg} / \mathrm{dL}, P=0.05)$ and a larger reduction in $\operatorname{ALT}(-11 \mathrm{U} / \mathrm{L}$ vs $-3 \mathrm{U} / \mathrm{L}, P=0.003$ ) (Figure 2 ).
Intention-to-treat analysis to evaluate impact of outcomes at week 12 demonstrated similar overall trends in change in weight, fasting glucose, TG, HDL and ALT (Table S3). There were more pronounced reductions in weight and resolution of low HDL among participants with NAFLD in this subanalysis. 


\subsection{Predictors of weight loss}

Characteristics of patients who did and those who did not achieve $\geq 5 \%$ weight reduction by programme completion are displayed in Table 3 . Those with $\geq 5 \%$ weight reduction were more likely to be women and to be white, and had a higher baseline weight (241 lb vs $235.5 \mathrm{lb}, P=0.05$ ) and a higher median ALT (35 vs $30 \mathrm{IU} / \mathrm{L}$, $P=0.03)$ compared to those with $<5 \%$ weight reduction. Likelihood of achieving $\geq 5 \%$ weight reduction was similar in patients with or without NAFLD. The most striking variable associated with achieving $\geq 5 \%$ weight reduction was duration of programme participation. As expected, individuals who were able to achieve $\geq 5 \%$ weight loss were more likely to have improvement in metabolic parameters including resolution of central obesity $(19.3 \%$ vs $6.9 \%, P=0.001)$ and impaired fasting glucose $(38.4 \%$ vs $23.2 \%, P=0.01)$, and more pronounced reductions in A1c, fasting glucose, fasting insulin, insulin resistance and TG. They also had more pronounced reduction in ALT.

A subanalysis of individuals with and without $\geq 10 \%$ weight loss noted that participants with a higher maximum weight $(P=0.04)$ and those who completed the 24-week programme were significantly more likely to achieve $\geq 10 \%$ weight loss $(82.7 \%$ vs $33.7 \%$, $P<0.001$ ). Participants who achieved $\geq 10 \%$ weight loss had higher rates of resolution of central obesity $(26.9 \%$ vs $9.5 \%, P=0.006)$, impaired fasting glucose $(76.5 \%$ vs $24 \%, P<0.001)$, insulin resistance $(70.6 \%$ vs $14.6 \%, P<0.001)$ and more pronounced improvements in A1c, TG and ALT (Table S4).

Multivariate analysis of predictors of $\geq 5 \%$ weight loss is demonstrated in Table S5. Duration of programme participation was the strongest independent predictor (odds ratio [OR] $6.7995 \% \mathrm{Cl}$ 3.712.4, $P<0.001$ ). White race was the only other significant variable with an OR of 3.83 (95\% Cl 1.04-14.03, $P=0.04$ ). Additional analysis of characteristics based on race was performed to evaluate whether any other covariates may be mediators of this relationship (Table S6). Participants of white race ( $\mathrm{N}=349,87 \%$ ) were older (55 vs 48.8 ), had less college/post-graduate education (68.6\% vs $88.6 \%$ ), a higher prevalence of HTN (66.5\% vs $48.8 \%$ ), larger waist circumference (46 vs 43 in), higher percentage body fat (45.5\% vs $43 \%$ ) and higher TG (160 vs 123) at baseline compared to participants of other races.

\section{4 | DISCUSSION}

Non-alcoholic fatty liver disease is one of the most common forms of chronic liver disease, affecting $25 \%-30 \%$ of the United States' population. ${ }^{18,19}$ Weight loss through lifestyle changes is a proven therapy for this highly prevalent disease. Unfortunately, implementation of lifestyle changes and achievement of sustained weight loss remains challenging for many patients. Structured lifestyle programmes often result in higher rates of weight loss due to regimented recommendations and follow-up. These types of programmes have long been designed and targeted for patients with cardiac or endocrine disease, but due to the underlying metabolic nature of NAFLD, lifestyle programmes are also relevant for patients with NAFLD.

The results of our study mirror findings of other investigations of prevalence of NAFLD among patients with metabolic disease. ${ }^{1,20}$ In this cohort, just under half of the patients had evidence of NAFLD. This is likely an underestimate as not all patients had undergone testing to evaluate for possible NAFLD. Another finding of note is that only one quarter of patients with NAFLD were referred to and managed by a GI or liver specialist for their NAFLD. Also of note, an additional one third of NAFLD patients were under the care of a GI provider for other conditions (eg, acid reflux and diarrhoea), but they did not receive care related to NAFLD. Taken together, this pattern of care reflects low disease awareness among patients and providers, even in the face of multiple metabolic risk factors. ${ }^{21,22}$ These care patterns also reflect perceptions regarding the clinical significance and treatment options for NAFLD held by many providers. ${ }^{23}$ This low rate of referral to $\mathrm{Gl} / \mathrm{Hepatology}$ is in line with data from prior studies where $71 \%$ of providers reported that they did not refer patients with suspected NAFLD to GI/Hepatology. ${ }^{24,25}$ These deficiencies in the care cascade represent key areas for improvement in order to optimise long-term outcomes for this burgeoning patient population. It is important to note the potential implications of low rates of referral including the potential to make accurate diagnoses of NAFLD and NASH and risk stratification for stage of liver disease. Although routine screening among high-risk patients is not currently recommended due to uncertainties around diagnostic approaches and cost-effectiveness of screening, the role for screening will continue to evolve as additional therapeutic options and predictive models for advanced or rapidly progressive NAFLD become available. ${ }^{26-}$ 28

We also highlighted the differences in baseline characteristics of patients with and without NAFLD among patients with the MetSyn. Patients with NAFLD were younger and more likely to have higher baseline weight, maximum lifetime weight and central obesity. They were also more likely to have hyperTG, low HDL-C and higher HOMA-IR. These unique characteristics underscore the importance of central adiposity and insulin resistance in NAFLD as metabolic health has been independently associated with risk of NASH and significant fibrosis. ${ }^{29,30}$ Despite having more pronounced metabolic derangements at baseline, NAFLD patients were equally likely to respond to the lifestyle programme. This finding is of note given concerns that NAFLD patients may have different uptake and response rates to lifestyle changes stemming from different baseline motivation to change and lower confidence to exercise. ${ }^{31-33}$ In this study, we found that duration of programme participation was the strongest predictor of weight loss. In our prior studies, we had demonstrated that the number of MetS criteria was correlated with the number of programme sessions attended (OR 2.27), highlighting a potential predictor of programme completion that can be used to help target interventions to increase adherence among participants. ${ }^{34}$ Although race was an independent predictor of weight loss, we are limited in our ability to draw conclusions from this finding 
TAB LE 3 Baseline characteristics and change in metabolic, liver-related and depression parameters stratified by achievement of $\geq 5 \%$ weight loss

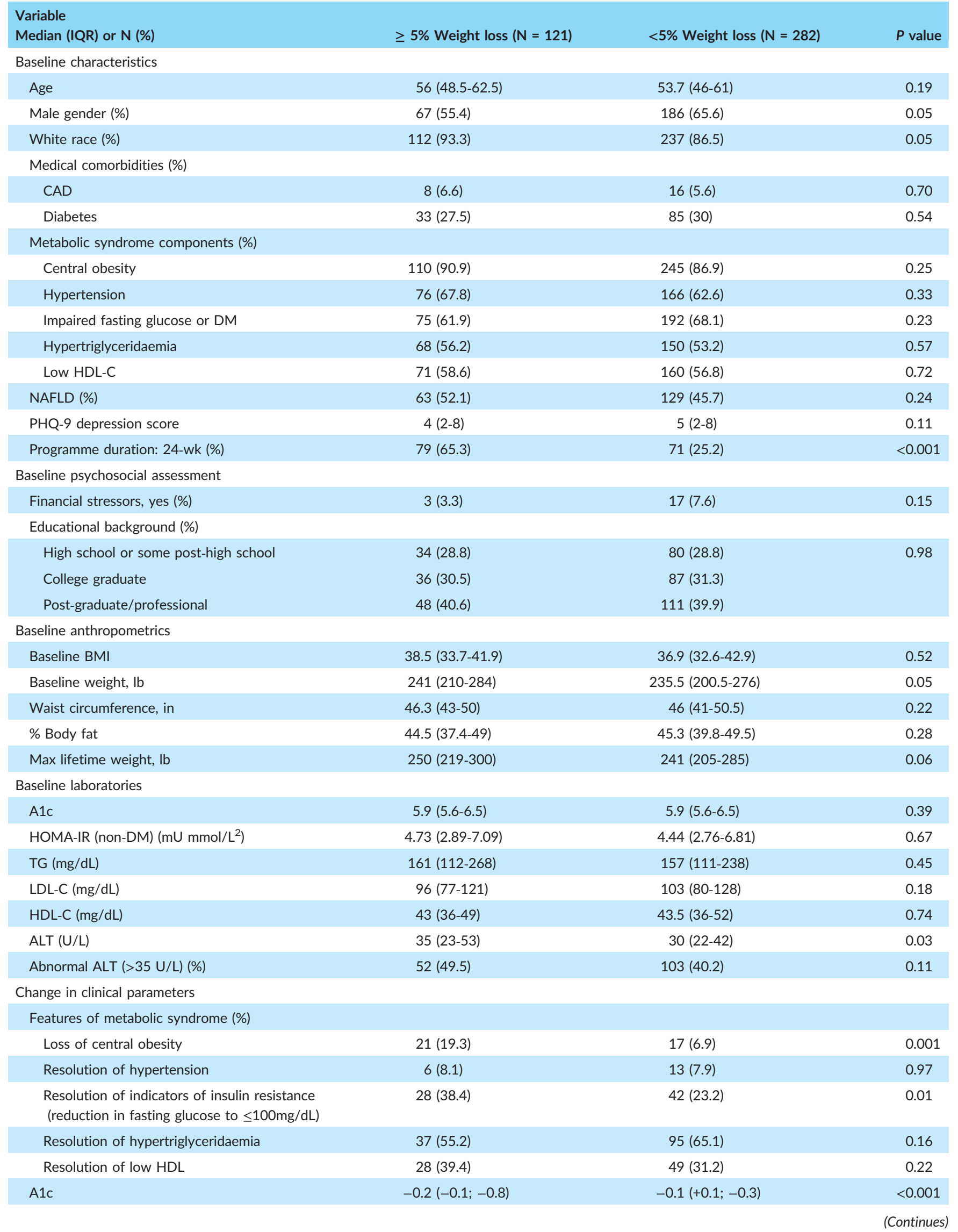


TABLE 3 (Continued)

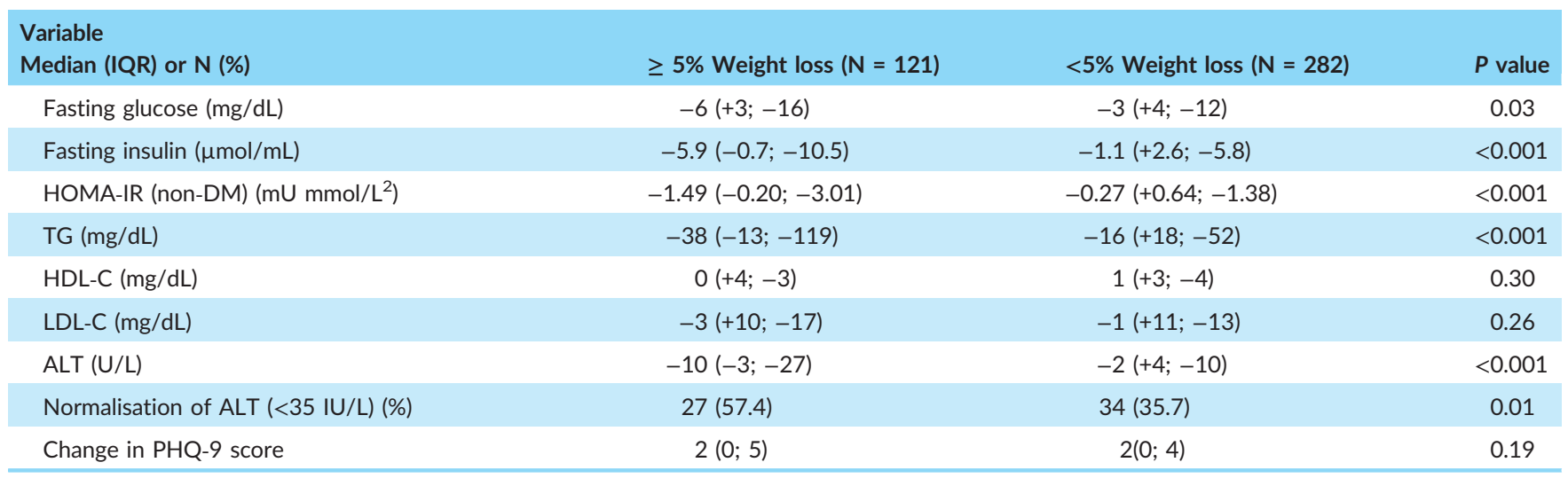

Central obesity defined as $\geq 40$ inches in men and $\geq 35$ inches in women. Hypertension defined as SBP $>130 \mathrm{~mm} \mathrm{Hg}$, DBP $>85 \mathrm{~mm} \mathrm{Hg}$ or on antihypertensive. Impaired fasting glucose defined as $>100 \mathrm{mg} / \mathrm{dL}$ or DM; hyperTG defined as $\geq 150 \mathrm{mg} / \mathrm{dL}$ or on treatment for triglycerides. Low HDL-C defined as $<40 \mathrm{mg} / \mathrm{dL}$ men and $<50 \mathrm{mg} / \mathrm{dL}$ women.

ALT, alanine aminotransferase; BMI, body mass index; HDL-C, high-density lipoprotein-cholesterol; HOMA-IR, homeostasis model assessment-estimated insulin resistance; LDL-C, low-density lipoprotein-cholesterol; TG, triglycerides.

given that $88 \%$ of participants were of white race. However, this finding has been previously described in the literature with higher rates of success in weight loss in part attributed to differences in basal metabolic rates. ${ }^{35-37}$ On subanalysis, those of non-white race appeared to have more favourable baseline metabolic characteristics (younger age, lower baseline waist circumference, percentage body fat and TG). These racial differences for propensity for weight loss are particularly interesting given the variable prevalence rates of NAFLD across different races and ethnicities. ${ }^{38,39}$

There are several limitations to note for our study. First, the diagnosis of NAFLD was made based on chart review and natural language processing and is inherently prone to both over- and underclassification. Second, our patient population is relatively homogenous and thus the results of this study may not generalise to the larger population of patients with NAFLD. Additionally, our current programme design does not include structured, prospective follow-up post-programme completion, so we are unable to speak to the durability of the benefits achieved through this programme. Our recent retrospective study of participants with follow-up data available in our health system $(\mathrm{N}=225)$ demonstrated that at 12 months after programme completion, while the majority of parameters were still improved compared to baseline, only change in LDL-C remained significantly improved compared to end of 12 -week programme. ${ }^{40}$ These findings echo those reported in follow-up after completion of most structured lifestyle intervention programmes where participants regain weight over time, but the majority are able to remain below programme entry weight. ${ }^{41-43}$ While programme design varies widely in terms of duration and intensity of follow-up, most programmes are designed for 3-6 months and do not incorporate post-programme follow-up. Overall, these findings of difficulty with maintenance of benefit post-programme completion highlight the primary limitation of these interventions and the need for maintenance interventions over the long term. ${ }^{44}$ Incorporation of follow-up touch points using eHealth modalities has been shown to be a potentially beneficial mechanism to sustain improvements over time, and as such warrant further investigation. ${ }^{45} \mathrm{~A}$ strength of our study in terms of NAFLD prevalence assessment was our ability to rule out heavy alcohol use in this cohort as an alcohol use assessment was performed as a standard part of enrolment into the programme. Another key strength is the detailed clinical, anthropometric and HRQOL assessments at baseline and programme completion that enabled us to conduct indepth analysis of the different types of benefits of fitness programmes in patients with MetSyn and NAFLD. While weight loss and improvements in metabolic parameters have been reported in many lifestyle intervention programmes, ours is one of the few demonstrating a decrease in PHQ-9, a measure of depression.

In conclusion, we demonstrated that roughly one-half of patients participating in a structured lifestyle intervention programme predominantly referred because of cardiovascular or endocrine diseases had evidence of underlying NAFLD, but only a quarter of the NAFLD patients were being specifically managed for their NAFLD by a specialist. We also found that metabolic derangements were more pronounced at baseline in NAFLD patients, but despite this NAFLD patients were equally responsive to the lifestyle programme. As expected, we found that a longer duration of programme participation was the most important predictor of significant weight loss. Our data can be used to help counsel NAFLD patients regarding expectations and timeline for lifestyle interventions to achieve target weight loss. It can also provide a framework for the design of future lifestyle intervention programmes targeted specifically for NAFLD patients. Future prospective assessment of for baseline NAFLD with detailed liver-specific endpoints including elastography would add to our knowledge of the disease-specific benefits of structured lifestyle programmes for this population.

\section{ACKNOWLEDGEMENT}

Declaration of personal and funding interests: None. 


\section{AUTHORSHIP}

\section{Guarantor of the article: Monica A. Konerman.}

Author contributions: MAK study design, analysis and drafting of manuscript. PW provision of data. MJ review of manuscript. EAJ review of manuscript. ASL study design and review of manuscript. MR data collection, study design and critical review of manuscript. All authors approved the final version of the manuscript.

\section{ORCID}

Monica A. Konerman (D) http://orcid.org/0000-0002-8381-6149

\section{REFERENCES}

1. Chen SH, He F, Zhou HL, Wu HR, Xia C, Li YM. Relationship between nonalcoholic fatty liver disease and metabolic syndrome. J Digest Dis. 2011;12:125-130.

2. Hamaguchi M, Kojima T, Takeda N, et al. The metabolic syndrome as a predictor of nonalcoholic fatty liver disease. Ann Intern Med. 2005;143:722-728.

3. Musso G, Gambino R, Cassader M, Pagano G. Meta-analysis: natural history of non-alcoholic fatty liver disease (NAFLD) and diagnostic accuracy of non-invasive tests for liver disease severity. Ann Med. 2011;43:617-649.

4. Younossi ZM, Koenig AB, Abdelatif D, Fazel Y, Henry L, Wymer M. Global epidemiology of nonalcoholic fatty liver disease - meta-analytic assessment of prevalence, incidence, and outcomes. Hepatology. 2016;64:73-84

5. Promrat K, Kleiner DE, Niemeier HM, et al. Randomized controlled trial testing the effects of weight loss on nonalcoholic steatohepatitis. Hepatology. 2010;51:121-129.

6. Tamura Y, Tanaka Y, Sato F, et al. Effects of diet and exercise on muscle and liver intracellular lipid contents and insulin sensitivity in type 2 diabetic patients. J Clin Endocrinol Metabol 2005;90:31913196.

7. Rubenfire M, Mollo L, Krishnan S, et al. The metabolic fitness program: lifestyle modification for the metabolic syndrome using the resources of cardiac rehabilitation. J Cardiopulm Rehabil Prevent. 2011;31:282-289.

8. National Cholesterol Education Program (NCEP) Expert Panel on Detection, Evaluation, and Treatment of High Blood Cholesterol in Adults (Adult Treatment Panel III). Third Report of the National Cholesterol Education Program (NCEP) Expert Panel on detection, evaluation, and treatment of high blood cholesterol in adults (Adult Treatment Panel III) final report. Circulation. 2002;106:3143-3421.

9. Walden $\mathrm{P}$, Jiang $\mathrm{Q}$, Jackson EA, Oral EA, Weintraub MS, Rubenfire $M$. Assessing the incremental benefit of an extended duration lifestyle intervention for the components of the metabolic syndrome. Diabetes Metab Syndr Obes. 2016;9:177-184.

10. Borg G. Borg's Perceived Exertion and Pain Scales. Champaign, IL: Human Kinetics; 1998.

11. American College of Sports M; Thompson WR, Gordon NF, Pescatello LS. ACSM's Guidelines for Exercise Testing and Prescription. Philadelphia, PA: Wolters Kluwer/Lippincott Williams \& Wilkins; 2010.

12. Ehrman J, Visich P, Keteyian S. Clinical Exercise Physiology (4th edn). Champaign, IL: Human Kinetics Publisher; 2018.

13. Frankenfield D, Roth-Yousey L, Compher C. Comparison of predictive equations for resting metabolic rate in healthy nonobese and obese adults: a systematic review. J Am Diet Assoc. 2005;105:775789.
14. Kroenke K, Spitzer RL, Williams J. The PHQ-9: validity of a brief depression severity measure. J Gen Intern Med. 2001;16:606-613.

15. Hanauer DA, Mei Q, Law J, Khanna R, Zheng K. Supporting information retrieval from electronic health records: A report of University of Michigan's nine-year experience in developing and using the Electronic Medical Record Search Engine (EMERSE). J Biomed Inform. 2015;55:290-300. PMID: 25979153.

16. Qu H-Q, Li Q, Rentfro AR, Fisher-Hoch SP, McCormick JB. The definition of insulin resistance using HOMA-IR for Americans of Mexican descent using machine learning. PLoS ONE. 2011;6:e21041.

17. Angulo $P$, Hui JM, Marchesini $G$, et al. The NAFLD fibrosis score: a noninvasive system that identifies liver fibrosis in patients with NAFLD. Hepatology. 2007;45:846-854.

18. Vernon G, Baranova A, Younossi ZM. Systematic review: the epidemiology and natural history of non-alcoholic fatty liver disease and non-alcoholic steatohepatitis in adults. Aliment Pharmacol Ther. 2011;34:274-285.

19. Wong RJ, Liu B, Bhuket T. Significant burden of nonalcoholic fatty liver disease with advanced fibrosis in the US: a cross-sectional analysis of 2011-2014 National Health and Nutrition Examination Survey. Aliment Pharmacol Ther. 2017;46:974-980.

20. Gerber L, Otgonsuren M, Mishra A, et al. Non-alcoholic fatty liver disease (NAFLD) is associated with low level of physical activity: a population-based study. Aliment Pharmacol Ther. 2012;36:772-781.

21. Wieland AC, Mettler P, McDermott MT, Crane LA, Cicutto LC,BambhaKM. Low awareness of nonalcoholic fatty liver disease among patients at high metabolic risk. J Clin Gastroenterol. 2014;49:e6-e10.

22. Ghevariya V, Sandar N, Patel K, et al. Knowing what's out there: awareness of non-alcoholic fatty liver disease. Front Med. 2014;1:4.

23. Wieland AC, Quallick M, Truesdale A, Mettler P, Bambha KM. Identifying practice gaps to optimize medical care for patients with nonalcoholic fatty liver disease. Dig Dis Sci. 2013;58:2809-2816.

24. Bergqvist CJ, Skoien R, Horsfall L, Clouston AD, Jonsson JR, Powell EE Awareness and opinions of non-alcoholic fatty liver disease by hospital specialists. Intern Med J. 2013;43:247-253.

25. Said A, Gagovic V, Malecki K, Givens ML, Nieto FJ. Primary care practitioners survey of non-alcoholic fatty liver disease. Ann Hepatol. 2013;12:758-765.

26. Chalasani N, Younossi Z, Lavine JE, et al. The diagnosis and management of nonalcoholic fatty liver disease: practice guidance from the American Association for the Study of Liver Diseases. Hepatology. 2018;67:328-357.

27. Corey KE, Klebanoff MJ, Tramontano AC, Chung RT, Hur C. Screening for nonalcoholic steatohepatitis in individuals with type 2 diabetes: a cost-effectiveness analysis. Dig Dis Sci. 2016;61:2108-2117.

28. Wong VW, Chalasani N. Not routine screening, but vigilance for chronic liver disease in patients with type 2 diabetes. J Hepatol. 2016;64:1211-1213.

29. Pang Q, Zhang J-Y, Song S-D, et al. Central obesity and nonalcoholic fatty liver disease risk after adjusting for body mass index. World $J$ Gastroenterol. 2015;21:1650-1662.

30. Ampuero J, Aller R, Gallego-Durán R, et al. The effects of metabolic status on non-alcoholic fatty liver disease-related outcomes, beyond the presence of obesity. Aliment Pharmacol Ther. 2018. https://doi. org/10.1111/apt.15015 [Epub ahead of print].

31. Frith J, Day CP, Robinson L, Elliott C, Jones DE, Newton JL. Potential strategies to improve uptake of exercise interventions in non-alcoholic fatty liver disease. J Hepatol. 2010;52:112-116.

32. Centis E, Moscatiello S, Bugianesi E, et al. Stage of change and motivation to healthier lifestyle in non-alcoholic fatty liver disease. $J$ Hepatol. 2013;58:771-777.

33. Stewart KE, Haller DL, Sargeant C, Levenson JL, Puri P, Sanyal AJ. Readiness for behaviour change in non-alcoholic fatty liver disease: implications for multidisciplinary care models. Liver Int. 2015;35:936943. 
34. Walden $\mathrm{P}$, Jiang $\mathrm{Q}$, Jackson EA, Oral EA, Weintraub MS, Rubenfire $M$. Assessing the incremental benefit of an extended duration lifestyle intervention for the components of the metabolic syndrome. Diabet Metab Syndr Obes. 2016;9:177-184.

35. DeLany JP, Jakicic JM, Lowery JB, Hames KC, Kelley DE, Goodpaster $\mathrm{BH}$. African American women exhibit similar adherence to intervention but lose less weight due to lower energy requirements. Int J Obes. 2013;38:1147.

36. Gannon B, DiPietro L, Poehlman ET. Do African Americans have lower energy expenditure than Caucasians? Int J Obes Relat Metab Disord. 2000;24:4-13.

37. Davis KK, Tate DF, Lang W, et al. Racial differences in weight loss among adults in a behavioral weight loss intervention: role of diet and physical activity. J Phys Act Health. 2015;12:1558-1566.

38. Schneider A, Lazo M, Selvin E, Clark JM. Racial differences in nonalcoholic fatty liver disease in the U.S. population. Obesity (Silver Spring, MD). 2014;22:292-299.

39. Pan J-J, Fallon MB. Gender and racial differences in nonalcoholic fatty liver disease. World J Hepatol. 2014;6:274-283.

40. Joseph $M$, Zhang $M$, Wei $B$, et al. Long-term outcomes following completion of a structured nutrition and exercise lifestyle intervention program for patients with metabolic syndrome. Diabetes Metab Syndr Obes. 2018. accepted.

41. Dalzill C, Nigam A, Juneau M, et al. Intensive lifestyle intervention improves cardiometabolic and exercise parameters in metabolically healthy obese and metabolically unhealthy obese individuals. Can $J$ Cardiol. 2014;30:434-440.

42. Pettman TL, Buckley JD, Misan GM, Coates AM, Howe PR. Health benefits of a 4-month group-based diet and lifestyle modification program for individuals with metabolic syndrome. Obes Res Clin Pract. 2009;3:221-235.

43. Svetkey LP, Stevens VJ, Brantley PJ, et al. Comparison of strategies for sustaining weight loss: the weight loss maintenance randomized controlled trial. JAMA. 2008;299:1139-1148.

44. Colombo CM, de Macedo RM, Fernandes-Silva MM, et al. Shortterm effects of moderate intensity physical activity in patients with metabolic syndrome. Einstein (Sao Paulo, Brazil). 2013;11:324-330.

45. Wing RR, Tate DF, Gorin AA, Raynor HA, Fava JL. A self-regulation program for maintenance of weight loss. $N$ Engl J Med. 2006;355:1563-1571.

\section{SUPPORTING INFORMATION}

Additional supporting information will be found online in the Supporting Information section at the end of the article.

How to cite this article: Konerman MA, Walden P, Joseph M, Jackson EA, Lok AS, Rubenfire M. Impact of a structured lifestyle programme on patients with metabolic syndrome complicated by non-alcoholic fatty liver disease. Aliment Pharmacol Ther. 2019;49:296-307. https://doi.org/10.1111/ $\underline{\text { apt.15063 }}$ 